\title{
Dolor irruptivo oncológico inducido por radioterapia: ¿es posible optimizar su tratamiento?
}

L. Olay Gayosoํㅡ, G. J. Rijo², Á. Rodríguez Sánchez³, R. Pozas de Celis ${ }^{4}$, C. Ferrer Albiach ${ }^{5}$, A. J. Conde Moreno ${ }^{5}$, O. Antonini ${ }^{6}$, G. Triana Martínez ${ }^{7}$, P. Calvo Crespo ${ }^{8}$, M. López Muñoz ${ }^{9}$, M. A. Estornell Gualde ${ }^{9}$, J. L. Monroy Antón ${ }^{9}$, F. López Campos ${ }^{10}$, J. C. Martínez Cedrés ${ }^{11}$, L. Alled Comín ${ }^{12}$, G. Asín ${ }^{13}$, C. Victoria Fernández ${ }^{14}$, J. E. Castro Gómez ${ }^{14}$, V. Muñoz Garzón ${ }^{15}$ y M. Lanzuela ${ }^{16}$

Servicios de Oncología Radioterápica. ${ }^{1}$ Hospital Universitario Central de Asturias. Oviedo. ${ }^{2}$ Hospital Jove. Gijón. ${ }^{3}$ Hospital de Basurto. Bilbao, Vizcaya. ${ }^{4}$ Hospital de Txagorritxu. Vitoria-Gastéiz, Álava. ${ }^{5}$ Hospital de Castellón. Castellón de la Plana. ${ }^{6} \mathrm{Hospital}$ Universitario La Fe. Valencia. ${ }^{7}$ Centro Oncológico de Galicia. A Coruña. ${ }^{8}$ Hospital Universitario de Santiago. Santiago de Compostela, A Coruña. ${ }^{9}$ Hospital Universitario de la Ribera. Alzira, Valencia ${ }^{10}$ Hospital Quirón Torrevieja. Alicante. ${ }^{11}$ Hospital Nuestra Señora de la Candelaria. Santa Cruz de Tenerife ${ }^{12}$ Hospital Clínico Universitario Lozano Blesa. Zaragoza. ${ }^{13}$ Hospital de Navarra. Pamplona. ${ }^{14}$ Hospital Cristal del Complejo Hospitalario de Orense. Ourense.

${ }^{15}$ Hospital Mexoeiro de Vigo. Pontevedra. ${ }^{16} \mathrm{Hospital} \mathrm{Miguel} \mathrm{Servet.} \mathrm{Zaragoza}$

Olay Gayoso L, Rijo GJ, Rodríguez Sánchez A, Pozas de Celis $R$, Ferrer Albiach C, Conde Moreno AJ, Antonini O, Triana Martínez G, Calvo Crespo P, López Muñoz M, Estornell Gualde MA, Monroy Antón JL, López Campos F, Martínez Cedrés JC, Alled Comín L, Asín G, Victoria Fernández C, Castro Gómez JE, Muñoz Garzón V, Lanzuela M. Dolor irruptivo oncológico inducido por radioterapia: ¿es posible optimizar su tratamiento? Rev Soc Esp Dolor 2015;22(4):159-164.

\begin{abstract}
Introduction: In the context of radiotherapy, control of breakthrough cancer pain (BTPC) is particularly challenging. BTPc has been defined by the Spanish Society of Pain (SED), the Spanish Society of Medical Oncology (SEOM) and the Spanish Society for Palliative Care (SECPAL) as a sudden and transient exacerbation of pain of great intensity (VAS > 7) and short (less than 20-30 minutes), which appears on the basis of a stable persistent pain when it is reduced to a tolerable level (VAS $<5$ ) by using major opioids.

Objectives: The main objective of this study was to assess the intensity of BTPc induced by cancer treatments that included radiotherapy $(\mathrm{RT})$, both exclusive and associated with che-
\end{abstract}

Recibido: 12-08-14.

Aceptado: 15-10-14. motherapy (RT/CT). Secondly, the efficacy of treatment was evaluated with fentanyl sublingual scheduled for BTPc control.

Material and methods: Retrospective, observational study in 110 patients recruited in 19 Spanish Radiotherapy Services. Patients must have BTPc induced by RT or RT/CT, with or without medication prescribed and with an intensity outside a VAS $>6$ in the last 24-48 h. Controls were established at baseline and at 3, 7, 15 and 30 days.

Results: There was a decrease in mean values on the VAS scale as the study progressed (VAS $=6$ in the control 0 to VAS $=3$ in the control 3) and the differences were significant $(p<$ 0.0001 ). Treatment satisfaction was rated as good or excellent by $85.3 \%$ of patients and $92.7 \%$ of researches.

Conclusions: The results of this study demonstrate the efficacy of BTPc treatment with sublingual fentanyl in the context of the radiotherapy cancer treatment, with a significant decrease in VAS from baseline values. The high satisfaction among physicians and patients with this treatment reflects the efficacy and convenience of sublingual fentanyl in controlling BTPC.

Key words: Breakthrough cancer pain. Radiotherapy. Fentanyl sublingual.

\section{RESUMEN}

Introducción: en el contexto de la radioterapia, el control del dolor irruptivo oncológico (DIO) supone un reto especial. El DIO ha sido definido por la Sociedad Española del Dolor (SED), la Sociedad Española de Oncología Médica (SEOM) y la 
Rev. Soc. Esp. del Dolor, Vol. 22, N. ${ }^{\circ}$ 4, Julio-Agosto 2015

Sociedad Española de Cuidados Paliativos (SECPAL) como una exacerbación del dolor súbita y transitoria, de gran intensidad (EVA > 7) y de corta duración (inferior a 20-30 minutos), que aparece sobre la base de un dolor persistente estable cuando este se encuentra reducido a un nivel tolerable $(\mathrm{EVA}<5)$ mediante el uso de opioides mayores.

Objetivos: el objetivo principal de este estudio fue evaluar la intensidad del DIO inducido por tratamientos oncológicos que incluyeran radioterapia (RT), tanto exclusiva como asociada a quimioterapia (RT/QT). Secundariamente, se evaluó la eficacia del tratamiento con fentanilo sublingual pautado para el control del DIO.

Material y métodos: estudio observacional retrospectivo realizado en 110 pacientes reclutados en 19 Servicios de Radioterapia españoles. Los pacientes debían presentar DIO inducido por RT o RT/QT, con o sin medicación pautada y cuya intensidad fuera de una EVA $>6$ en las últimas 24-48 h. Se establecieron controles en el momento basal, y a los 3, 7, 15 y 30 días.

Resultados: se apreció un descenso en la media de los valores en la escala EVA según avanzó el estudio (EVA = 6 en el control 0 a EVA $=3$ en el control 3), y las diferencias fueron significativas $(p<0,0001)$. La satisfacción con el tratamiento fue calificada como buena o excelente por el $85,3 \%$ de los pacientes y por el $92,7 \%$ de los investigadores.

Conclusiones: los resultados de este estudio demuestran la eficacia del tratamiento del DIO con fentanilo sublingual en el contexto del tratamiento oncológico radioterápico, con un descenso significativo en los valores EVA frente al valor basal. La elevada satisfacción de los médicos y pacientes con este tratamiento refleja la eficacia y la comodidad del fentanilo sublingual en el control del DIO.

Palabras clave: Dolor irruptivo oncológico. Radioterapia. Fentanilo sublingual.

\section{INTRODUCCIÓN}

Más del 50\% de los pacientes con cáncer sufren dolor, habitualmente de intensidad moderada o intensa. En pacientes con enfermedad avanzada, el tratamiento del dolor supone un reto (1). Distintas guías recomiendan tratar el dolor persistente con terapia de base, por lo general con una dosis estable de opioides (2-4). A pesar del tratamiento, muchos pacientes siguen experimentando episodios agudos de dolor intenso, conocido como "dolor irruptivo oncológico" (DIO). Este se define como una exacerbación aguda del dolor de rápida aparición, corta duración y de moderada a elevada intensidad que sufre el paciente cuando presenta un dolor basal estabilizado y controlado con opioides (5). El DIO puede ser previsible o imprevisible y, según el motivo desencadenante, se clasifica como idiopático, incidental (originado por el movimiento) o debido al final de una dosis terapéutica (6).

En el año 2002, la Sociedad Española de Oncología Médica (SEOM), la Sociedad Española de Cuidados Paliativos (SECPAL) y la Sociedad Española del Dolor (SED) establecieron un consenso en el que asumieron el término "dolor irruptivo" para definir una exacerbación del dolor súbita y transitoria, de gran intensidad (EVA > 7) y de corta duración (inferior a 20-30 minutos), que aparece sobre la base de un dolor persistente estable cuando este se encuentra reducido a un nivel tolerable $(\mathrm{EVA}<5)$ mediante el uso de opioides mayores.

En cuanto a la prevalencia del DIO, esta se ha cifrado entre el 50-75\% de los pacientes oncológicos $(1,3,6)$. Sin embargo, en un estudio realizado en España con 397 pacientes se comunicó una prevalencia del $23 \%$ en los pacientes con cáncer avanzado, lo que probablemente refleje el infradiagnóstico de esta afección. Este estudio, asimismo, demostró que un $41 \%$ de los pacientes oncológicos sufre al menos un episodio de DIO al día (7).

En el contexto de la radioterapia, el control del DIO también supone un reto especial. Los pacientes sometidos a radioterapia pueden experimentar distintas situaciones de dolor previsible. Este puede deberse a la necesidad de permanecer inmovilizado durante la sesión de radioterapia, a la necesidad de llevar una máscara de inmovilización (cáncer de cabeza y cuello), a la odinofagia originada por la mucositis, a la defecación tras el desarrollo de proctitis, o al dolor súbito durante la noche que provoca alteraciones del sueño (8).

Las recomendaciones para tratar el DIO han incluido históricamente la adición de un opioide de acción rápida al tratamiento de base. Más recientemente, las guías han resaltado la utilidad de los fentanilos de acción rápida. Estos compuestos, al presentar un rápido inicio de acción y una duración corta, encajan con el perfil de un episodio típico de DIO $(2-4,8)$.

Con estos antecedentes, el objetivo principal de este estudio fue evaluar la intensidad del DIO inducido por tratamientos oncológicos que incluyeran radioterapia, tanto exclusiva como asociada a quimioterapia. Secundariamente, se evaluó la eficacia del tratamiento con fentanilo sublingual pautado para el control del DIO.

\section{MATERIAL Y MÉTODOS}

Estudio observacional retrospectivo realizado en 110 pacientes reclutados en 19 Servicios de Radioterapia representativos del territorio español. Los criterios de inclusión fueron: pacientes ambulatorios mayores de 18 años, con diagnóstico de cáncer (cualquier localización) con independencia del estadio, que estuvieran recibiendo tratamiento con radioterapia (RT) o radioterapia/quimioterapia (RT/QT). Además, debían presentar DIO inducido por los tratamientos, con o sin medicación pautada y cuya intensidad fuera de una EVA $>6$ en las últimas 24-48 h.

Para cada paciente del estudio se recogieron como datos iniciales (control 0) el tratamiento analgésico basal, el tipo 
de DIO (idiopático o incidental, previsible o imprevisible), el valor medio en la escala EVA durante los episodios de DIO, los efectos secundarios al tratamiento analgésico basal, el número de episodios de DIO y la administración de medicación coadyuvante. En el resto de controles se registraron los siguientes datos: dosis media de fentanilo por episodio, valor medio en la escala EVA durante los episodios de DIO, efectos secundarios al tratamiento analgésico, número de episodios de DIO y administración de medicación coadyuvante.

En cuanto al esquema de visitas y controles, se realizó un control basal y posteriormente otros el día 3 (control 1), el día 7 (control 2), el día 15 (control 3) y el día 30 (control 4).

\section{Análisis estadístico}

Las variables cuantitativas fueron descritas mediante: $\mathrm{n}$ (número de observaciones disponibles), $\mathrm{n}$ ausentes (número de observaciones no disponibles), media, desviación típica, IC 95\% (intervalo de confianza de la media al 95\%), mediana y rango (valor mínimo y máximo). Las variables cualitativas fueron descritas mediante la frecuencia y el porcentaje. Los datos ausentes fueron descritos con su frecuencia pero no se incluyeron en el cálculo de los porcentajes.

Para analizar los distintos parámetros se utilizó la prueba ANOVA para análisis entre-grupos y la t de Student para muestras pareadas para análisis intragrupo en variables numéricas. Para las variables ordinales se utilizó el test de Chi-Cuadrado o test de Fisher.

Las tablas y análisis se realizaron con el programa estadístico SAS System 9.2.

\section{RESULTADOS}

\section{Características basales}

El $67,3 \%$ de los pacientes de este estudio $(n=110)$ eran hombres y el $32,7 \%$ mujeres. La edad media de los pacientes fue de 63 años (rango 26-84).

Tan sólo el 3,0\% de los pacientes sufrieron efectos secundarios con la medicación anterior al estudio. El esquema terapéutico principal más aplicado fue el concomitante (51,9\% de los pacientes), seguido de radioterapia exclusiva $(37,7 \%)$. En cuanto a la etiología del DIO, la causa observada con mayor frecuencia fue la mucositis $(55,2 \%)$, seguida de esofagitis (17,7\%) (Fig. 1).

La dosis basal de fentanilo sublingual fue de $100 \mathrm{mcg}$. En caso de que a los 30 minutos no se diera efecto analgésico, se añadieron otros $100 \mathrm{mcg}$.

\section{Objetivo principal}

En la escala EVA se apreció un descenso en la media de los valores según avanzó el estudio (de un valor de 6 en el control 0 a un valor 3 en el control 3). Se encontraron diferencias significativas en los cambios de todos los controles respecto al valor basal ( $\mathrm{p}<0,0001)$ (Fig. 2).

El tiempo de inicio de alivio del dolor en los distintos controles del estudio fue muy similar, y no se detectaron diferencias estadísticamente significativas entre ellos ( $\mathrm{p}=$ 0,7858).

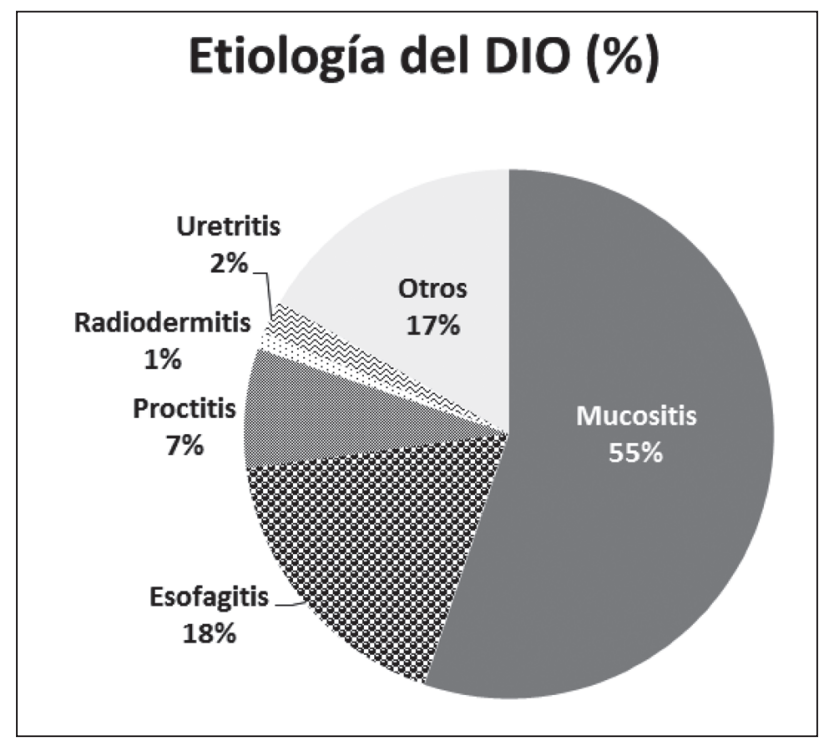

Fig. 1. Disposición de los pacientes $(n=110)$ según la causa del dolor irruptivo oncológico (DIO).

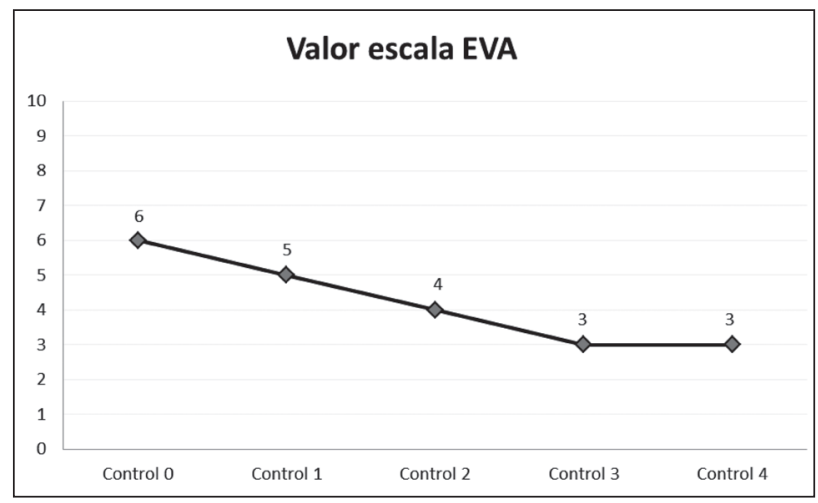

Fig. 2. Valoración en la escala visual analógica EVA (110) en los controles del estudio ( $p<0,0001$ en todos los controles). 


\section{Acontecimientos adversos}

El porcentaje de pacientes con al menos un efecto secundario fue similar en todos los controles del estudio $(45,0 \%$ en el control $0 ; 50,0 \%$ en el control $1 ; 50,4 \%$ en el control $2,49,1 \%$ en el control 3 y $38,3 \%$ en el control 4 ), y no se encontraron diferencias significativas entre dichos controles o visitas ( $\mathrm{p}=0,2946)$.

Al analizar las posibles diferencias de cada uno de los efectos entre los distintos controles, se encontraron diferencias significativas en términos de vómitos $(\mathrm{p}=0,0481$, más frecuentes en el control 0 ), y en alteraciones dermatológicas ( $\mathrm{p}=0,0013$, más frecuentes en el control 0).

\section{Medicación coadyuvante}

No se encontraron diferencias significativas en el uso de medicación coadyuvante en los distintos controles del estudio $(\mathrm{p}=0,1389)$.

\section{Grado de satisfacción del paciente y del investigador}

El porcentaje de pacientes cuyo grado de satisfacción fue "regular", "bueno" o "excelente" fue del 14,7\%, 51,0\% y $34,3 \%$, respectivamente. En cuanto al grado de satisfacción de los investigadores, este fue calificado como "regular" por el 7,3\%, "bueno" por el 54,2\% y "excelente" por el 38,5\% de ellos (Fig. 3).

\section{DISCUSIÓN}

El estudio del DIO ha cobrado relevancia en los últimos años, dada su estrecha relación con una peor calidad de vida del paciente oncológico y por ser una entidad poco conocida e infradiagnosticada (9-11). Además del sufrimiento que conllevan, los episodios de DIO generan ansiedad y añaden incapacidad funcional, lo cual se traduce en una mayor dificultad para controlar el dolor basal del paciente.

En una encuesta llevada a cabo en un Servicio de Radioterapia Oncológica, más de la mitad de los pacientes experimentaron dolor, y un 39\% de ellos indicaron que este no se trataba de forma adecuada $(12,13)$. Esta situación podría aumentar la ansiedad del paciente y provocar que desconfíe del tratamiento radioterápico o incluso que lo abandone. Así, uno de los motivos descritos para explicar los abandonos del tratamiento radioterápico es la incapacidad de soportar los efectos adversos de la radioterapia $(14,15)$. Se ha notificado que esta falta de control del dolor puede por tanto reducir los posibles beneficios de la radioterapia (15-23).

Estas interrupciones del tratamiento y la prolongada duración de la radioterapia pueden resultar en una pérdida

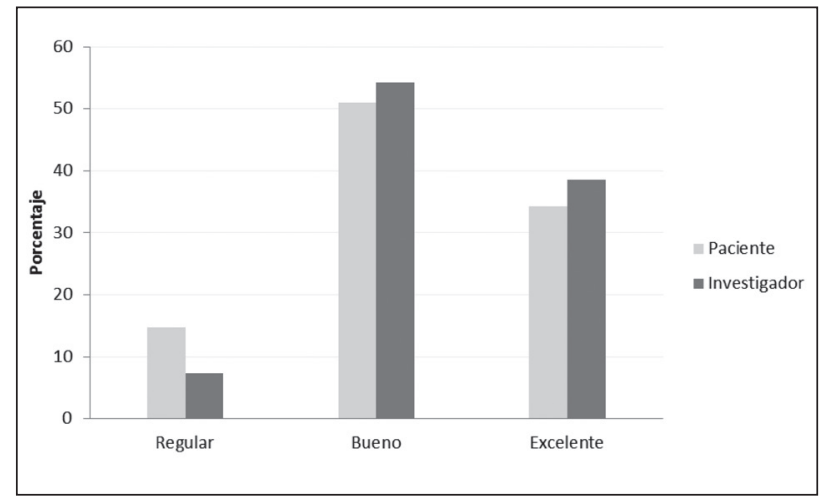

Fig. 3. Grado de satisfacción (\%) de los pacientes y de los investigadores del tratamiento con fentanilo sublingual en la última visita del estudio (control 4).

de control del tumor local con el consiguiente riesgo de metástasis. Este fenómeno se encuentra bien documentado en pacientes con carcinoma de células escamosas de cabeza y cuello, así como en otros tipos de cáncer (16-19,24). Así, se ha estimado que la tasa de repoblación durante un cese del tratamiento es casi cuatro veces más rápida que durante los días de irradiación (20,21). Asimismo, se ha estimado que al alargar el tiempo de radioterapia en un día el control local se reduce en un 1,4-1,7\% cada día $(16,22)$. En otro estudio se estimó que, por cada día de interrupción de la radioterapia, la tasa de fracaso terapéutico se incrementó en un 3,3\% para el control del tumor local y regional, y en un 2,9\% para la supervivencia libre de progresión (15).

Debe resaltarse que el manejo adecuado del DIO se basa en tres aspectos: prevención, anticipación y empleo de medicación adecuada. Además, es clave hacer una adecuada titulación de la analgesia de base y dejar prescrita de antemano medicación "de rescate" con opioides de liberación rápida, que serán administrados además del tratamiento opioide de base en caso de aparición del dolor (25).

El DIO previsible podría estar especialmente asociado al proceso radioterápico. Los pacientes deben permanecer inmovilizados durante las sesiones, en ocasiones deben llevar una máscara inmovilizadora; además la radioterapia se asocia a la aparición de mucositis y proctitis que cursan con dolor. En estas situaciones, la previsión de una medicación de rescate podría reducir el riesgo de ansiedad y de abandono del tratamiento por parte del paciente. En caso de elegir un opioide de acción corta, habría que administrarlo al menos una hora antes del episodio, lo cual suele ser poco práctico $(2,26)$. En cambio, un fentanilo de acción rápida, con un rápido inicio de acción, podría administrarse minutos antes del dolor previsto $(4,27,28)$.

El perfil farmacocinético óptimo del fármaco destinado a controlar los episodios de DIO sería, por tanto, aquel con una alta potencia, un rápido inicio de acción y una corta 
duración, y el que más se ajusta a estas características, a día de hoy, es el fentanilo (25). Hasta ahora se ha venido utilizando la vía oral por su alta permeabilidad, lipofilia, mayor biodisponibilidad y comodidad. Como alternativa a esta vía se dispone actualmente del citrato de fentanilo por vía sublingual, que puede presentar ciertas ventajas al ser más permeable y más rápida, además de evitar la pérdida de fármaco con la ingestión (25).

\section{CONCLUSIONES}

Los resultados de este estudio demuestran la eficacia del tratamiento del DIO con fentanilo sublingual en el contexto del tratamiento oncológico radioterápico, con un descenso significativo en los valores EVA frente al valor basal.

La satisfacción con el tratamiento fue evaluada como buena o excelente por el $85,3 \%$ de los pacientes y por el $92,7 \%$ de los médicos, lo que refleja la eficacia y la comodidad del fentanilo sublingual en el control del DIO.

Estas experiencias apuntan a la necesidad de seguir investigando, mediante ensayos clínicos controlados, en la optimización del control del DIO en el contexto del tratamiento radioterápico del paciente oncológico.

\section{AGRADECIMIENTOS}

Los autores agradecen a GORNOR (Grupo de Oncología Radioterápica del Norte) y el apoyo recibido para la realización de este trabajo.

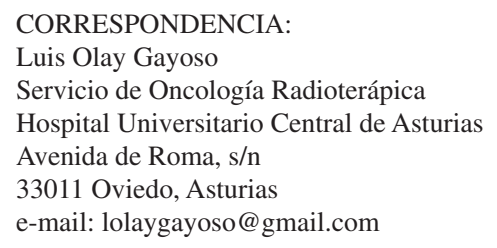

\section{BIBLIOGRAFÍA}

1. Van den Beuken-van Everdingen MH, de Rijke JM, Kessels AG, Schouten HC, van Kleef M, Patijn J. Prevalence of pain in patients with cancer: A systematic review of the past 40 years. Ann Oncol 2007;18:1437-49.

2. National Comprehensive Cancer Network: NCCN Clinical Practice Guidelines in Oncology (NCCN Guidelines): Adult Cancer Pain. Versión 1.2013. 2013. Disponible en: http:// www.nccn.org/professionals/physician_gls/f_guidelines. asp. Último acceso julio 2014.

3. Caraceni A, Davies A, Poulain P, Cortés-Funes H, Panchal J, Fanelli G. Guidelines for the management of breakthrough pain in patients with cancer. J Natl Compr Canc Netw 2013;11:S29-S36.
4. Caraceni A, Hanks G, Kaasa S, et al. Use of opioid analgesics in the treatment of cancer pain: Evidence-based recommendations from the EAPC. Lancet Oncol 2012;13:e58-e68.

5. Davies AN, Dickman A, Reid C, Stevens AM, Zeppetella G; Science Committee of the Association for Palliative Medicine of Great Britain and Ireland. The management of cancer-related breakthrough pain: Recommendations of a task group of the Science Committee of the Association for Palliative Medicine of Great Britain and Ireland. Eur J Pain 2009;13:331-8.

6. Portenoy RK, Hagen NA. Breakthrough pain: Definition, prevalence and characteristics. Pain 1990;41:273-81.

7. Gómez-Batiste X, Madrid F, Moreno F, Gracia A, Trelis J, Nabal M, et al. Breakthrough cancer pain: Prevalence and characteristics in patients in Catalonia, Spain. J Pain Symptom Manage 2002;24(1):45-52.

8. Bell BC, Butler EB. Management of predictable pain using fentanyl pectin nasal spray in patients undergoing radiotherapy. J Pain Res 2013;6:843-8.

9. Toledo Enes Nogueira R, Brandao de Moraes Vieira E, Albuquerque Sousa LH, et al. Difficult situation in cancer pain: Breakthrough pain. Rev Dor São Paulo 2014;15(1): 41-7.

10. Zeppetella G. Breakthrough pain in cancer patients. Clin Oncol (R Coll Radiol) 2011;23(6):393-8.

11. Haugen DF, Hjermstad MJ, Hagen N, Caraceni A, Kaasa $\mathrm{S}$, (EPCRC) EPCRC. Assessment and classification of cancer breakthrough pain: A systematic literature review. Pain 2010;149(3):476-82.

12. Pignon T, Fernandez L, Ayasso S, Durand M, Badinand D, Cowen D. Impact of radiation oncology practice on pain: a cross-sectional survey. Int J Radiat Oncol Biol Phys 2004;60:1204-10.

13. Janjan N. Do we need to improve pain management in the radiation oncology department? Nat Clin Pract Oncol 2005;2:130-1.

14. Chen YP, Tseng NM, Tsang CK, Lin SY. Causes of interruption of radiotherapy in nasopharyngeal carcinoma patients in Taiwan. Jpn J Clin Oncol 2000;30:230-4.

15. Kwong DL, Sham JS, Chua DT, Choy DT, Au GK, Wu PM. The effect of interruptions and prolonged treatment time in radiotherapy for nasopharyngeal carcinoma. Int J Radiat Oncol Biol Phys 1997;39:703-10.

16. Garau MM, Monné JS, Serés MJC, Binefa CM, Llopis MP. Compliance to the prescribed overall treatment time (OTT) of curative radiotherapy in normal clinical practice and impact on treatment duration of counteracting short interruptions by treating patients on Saturdays. Clin Transl Oncol 2009;11:302-11.

17. Dale RG, Hendry JH, Jones B, Robertson AG, Deehan C, Sinclair JA. Practical methods for compensating for missed treatment days in radiotherapy, with particular reference to head and neck schedules. Clin Oncol 2002;14:382-93.

18. Coles CE, Burgess L, Tan LT. An audit of delays before and during radical radiotherapy for cervical cancer-effect on tumour cure probability. Clin Oncol 2003;15:47-54.

19. D’Ambrosio DJ, Li T, Horwitz EM, Chen DYT, Pollack A, Buyyounouski MK. Does treatment interruption affect outcome after radiotherapy for prostate cancer? Int J Radiat Oncol Biol Phys 2008;72:1402-7.

20. Tarnawski R, Skladowski K, Swierniak A, Wygoda A, Mucha A. Repopulation of tumour cells during radiotherapy is doubled during treatment gaps. J Theor Med 2000;2:297305 . 
21. Tarnawski R, Fowler J, Skladowski K, Swierniak A, Suwiński R, Maciejewski B, et al. How fast is repopulation of tumor cells during the treatment gap? Int J Radiat Oncol Biol Phys 2002;54:229-36.

22. Bese NS, Hendry J, Jeremic B. Effects of prolongation of overall treatment time due to unplanned interruptions during radiotherapy of different tumor sites and practical methods for compensation. Int J Radiat Oncol Biol Phys 2007;68:654-61.

23. Kupelian P. Radiotherapy: Effect of treatment interruptions in prostate cancer. Nat Rev Clin Oncol 2009;6:312-3.

24. Russo G, Haddad R, Posner M, Machtay M. Radiation treatment breaks and ulcerative mucositis in head and neck cancer. Oncologist 2008;13:886-98.

25. Trinidad JM, Herrera J, Rodríguez MJ. Análisis de efectividad del citrato de fentanilo sublingual en pacientes con dolor irruptivo: estudio Sublime. Rev Soc Esp Dolor 2011; 18(4):207-18.

26. Murino P, Mammucari M, Borelli D, Pepe A, Giugliano MF, Morra A, et al. Role of immediate-release morphine (MIR) in the treatment of predictable pain in radiotherapy. J Pain Palliat Care Pharmacother 2011;25:121-4.

27. Fallon M, Reale C, Davies A, Lux AE, Kumar K, Stachowiak A, et al.; Fentanyl Nasal Spray Study 044 Investigators Group. Efficacy and safety of fentanyl pectin nasal spray compared with immediate-release morphine sulfate tablets in the treatment of breakthrough cancer pain: A multicenter, randomized, controlled, double-blind, double-dummy multiple-crossover study. J Support Oncol 2011;9:224-31.

28. Mercadante S. Pharmacotherapy for breakthrough cancer pain. Drugs 2012;72:181-90. 